\title{
Mimicking bug-like surface structures and their fluid transport produced by ultrashort laser pulse irradiation of steel
}

S. V. Kirner ${ }^{1, a}$, U. Hermens ${ }^{2}$, A. Mimidis ${ }^{3}$, E. Skoulas ${ }^{3}$, C. Florian ${ }^{4}$, F. Hischen ${ }^{5}$, C. Plamadeala ${ }^{5}$, W. Baumgartner ${ }^{5}$, K. Winands ${ }^{2}$, H. Mescheder ${ }^{2}$, J. Krüger ${ }^{1}$, J. Solis ${ }^{4}$, J. Siegel ${ }^{4}$, E. Stratakis ${ }^{3}$, J. Bonse ${ }^{1}$

${ }^{1}$ Bundesanstalt für Materialforschung und -prüfung (BAM), Unter den Eichen 87, D-12205 Berlin, Germany

${ }^{2}$ Fraunhofer Institute for Production Technology IPT, Steinbachstr. 17, D-52074 Aachen, Germany

${ }^{3}$ Institute of Electronic Structure and Laser, Foundation for Research and Technology - Hellas, Heraklion, GR71110 Crete, Greece

${ }^{4}$ Laser Processing Group, Instituto de Optica - CSIC (IO-CSIC), Serrano 121, E-28006 Madrid, Spain

${ }^{5}$ Johannes Kepler University Linz, Altenberger Straße 69, A-4040 Linz, Austria

\begin{abstract}
Ultrashort laser pulses with durations in the fs- to ps-range were used for large area surface processing of steel aimed at mimicking the morphology and extraordinary wetting behaviour of bark bugs (Aradidae) found in nature. The processing was performed by scanning the laser beam over the surface of polished flat sample surfaces. A systematic variation of the laser processing parameters (peak fluence and number of effective pulses per spot diameter) allowed the identification of different regimes associated with characteristic surface morphologies (laser-induced periodic surface structures, i.e. LIPSS, grooves, spikes, etc.). Moreover, different laser processing strategies, varying laser wavelength, pulse duration, angle of incidence, irradiation atmosphere, and repetition rates, allowed to achieve a range of morphologies that resemble specific structures found on bark bugs. For identifying the ideal combination of parameters for mimicking bug-like structures, the surfaces were inspected by scanning electron microscopy. In particular, tilted micrometre-sized spikes are the best match for the structure found on bark bugs.

Complementary to the morphology study, the wetting behaviour of the surface structures for water and oil was examined in terms of philic/-phobic nature and fluid transport. These results point out a route towards reproducing complex surface structures inspired by nature and their functional response in technologically relevant materials.
\end{abstract}

This is a post-peer-review, pre-copyedit version of an article published in [insert journal title]. The final authenticated version is available online at: https://doi.org/10.1007/s00339-017-1317-3

PACS: 79.20.Ds

a) author to whom correspondence should be addressed;

Electronic mail: sabrina.kirner@bam.de 


\section{INTRODUCTION}

During millions of years of evolution and natural selection, nature developed and tested numerous solutions for problems regarding surface structure and chemistry and eventually, function. Therefore, mimicking nature is often the most convenient way of implementing a certain biological process in solving current technological problems [1].

In our research, we are interested in special wetting properties, and we draw our inspiration from the integument of bugs of the genus Dysodius, namely lunatus and magnus [see Fig. 1(a)], also known as bark or flat bugs. These representatives of the Aradidae family can be found in South- and Central American tropics, living mostly on and under the bark of trees and feeding themselves on the fungi growing on these trees [2-4]. Both D. lunatus and D. magnus have their bodies ornamented with two different types of microstructures, both used to facilitate liquid transportation [5]. The first type of the microstructures is located on the connexival plates, which are interconnected by the segmental sutures, thus acting as capillary-like channels. Upon contact with a droplet of water, the water spreads immediately on the connexival plates and is transported over the whole dorsal surface through capillary channels, showing a hemi-wicking wetting regime [6]. Located under the wings, Aradidae have a channel consisting of three scent gland openings [see Fig. 1(b), red rectangles] [7] for secreting an oily defensive liquid [8-10]. In contrast to the earlier mentioned hydrophilic micro-ornamented surface on the connexival plates, the surface under the wings is hydrophobic. The gland openings are surrounded by microstructures, which have the aim of facilitating directional movement of the oil from the glands to the wings' bases, where the oily defensive liquid is actually evaporated. Viewed from the top, the microstructures appear as caudally-oriented spikes [see Fig. 1(c)], with a length of $10.5 \mu \mathrm{m}$, width of $5.4 \mu \mathrm{m}$, height of $3.6 \mu \mathrm{m}$, and an average distance between two microstructures of $10 \mu \mathrm{m}$. The associated aspect ratios are as follow: length-to-width: $1.9: 1$, length-to-height: $2.9: 1$, and width-toheight: $1.5: 1$. In this case, the cuticle with the microstructures and the wing form a closed capillary channel and the directional transport takes place because of better pinning of the liquid front at the spikes' tips [11]. In related species, similarly micro-ornamented channels, the so called peritremes, can be found as part of the external scent efferent system, and have the same function of transporting the liquid directionally from the gland orifice to the evaporative areas [12].

In this study we tried to mimic the described hydrophobic microstructured surface found under the wings of $D$. magnus on an inorganic material. This was done by ultrashort-pulse laser processing of an alloyed steel (42CrMo4) with three different laser setups (different wavelengths, pulse durations and repetition rates), varying the irradiation parameters (peak fluence $\phi_{0}$, number of effective pulses $N_{\text {eff }}$ and irradiation angle) as well as the surrounding atmospheres. We obtained microstructures of different sizes and periodicities, which exhibit hydrophobic behaviour. To obtain a directional fluid transport of water as well as oily liquids we generated gradient structures. The chosen steel type is very common and features various industrial applications, for instance in technical components that suffer from friction and wear. Directional fluid transport on micro-patterned steel is of interest in the field of tribology, as it can support a reduction of friction and wear. Thus, our results on the laserstructured steel surfaces may contribute to new tribological applications, where directional liquid transport is required, for example in mechanical parts that are in motion.

\section{EXPERIMENTAL}

Nitrided 42CrMo4 (1.7225) alloyed steel samples with a diameter of 25 or $40 \mathrm{~mm}$ and a height of 9 or $12 \mathrm{~mm}$, respectively, were supplied by Miba Gleitlager GmbH (Laakirchen, Austria). The surfaces of the disk- shaped samples were polished resulting in a mirror-like surface finish with an average roughness $R_{\mathrm{a}}<10 \mathrm{~nm}$, which was measured by a surface profilometer (Hommel-Etamic TK100) over a distance of $1.5 \mathrm{~mm}$. 
Unless noted otherwise, the laser processing was performed in air by movement of either the focused laser beam or the sample relative to each other in a meandering way at constant scan velocity $v_{x}$ with a line offset $\Delta y$ - compare reference [13]. The laser systems at all institutions provide linearly polarized laser pulses. The processing parameters for each setup differ (see Tab. 1). For each laser processing system, the Gaussian beam waist measured at $1 / e^{2}$-radius $w_{0}$ of the focussed laser beam was individually determined at the sample surface using a method introduced by Liu [14]. The corresponding peak fluences (at the sample surface) were calculated from the laser pulse energy $E$ via $\phi_{0}=2 E /\left(\pi w_{0}^{2}\right)$ [15]. The effective number of laser pulses per focused laser spot diameter $D=2 w_{0}$ in $x$-direction accounts for $N_{\text {eff_1D }}=(D \times f) / v_{\mathrm{x}}$, depending on the laser pulse repetition rate $f[15]$.

At BAM, 25-mm diameter samples were mounted on a motorized 3-axis, linear translation stage and placed normal to the incident laser beam close to the focal position of a spherical dielectric mirror with a focal length of $500 \mathrm{~mm}$. At this sample position, a $1 / e^{2}$ beam waist diameter of $2 w_{0}=130 \mu \mathrm{m}$ (112 $\mu \mathrm{m}$ for the gradient structures) was measured for the focussed laser beam ( $\tau=30$ fs pulse duration). The laser radiation was emitted from a Ti:sapphire amplifier system (Compact Pro, Femtolasers, Vienna, Austria) operated at a central wavelength of $\lambda=790 \mathrm{~nm}$ and at $f=1 \mathrm{kHz}$ pulse repetition rate. Between laser processing and characterizations, the samples were stored in a desiccator. However, while being shipped between the institutes for wetting test and fluid transport measurements, the samples were exposed to air.

The laser processing setup used by the Fraunhofer IPT is depicted in Fig. 2. The sample was mounted on a 5-axis stage of a machine tool (Kern Microtechnik $\mathrm{GmbH}$, Eschenlohe, Germany) and was tilted by a certain angle with respect to the laser beam. The tilting axis is indicated in red in Fig. 2. Two different scanning directions (black) with respect to the tilting axis (red) were used.

Different surface morphologies were fabricated by linearly polarized picosecond laser pulses (Super Rapid, Lumera Laser GmbH, Kaiserslautern, Germany) with a pulse duration of $\tau=9 \mathrm{ps}$ and a wavelength of $\lambda=532 \mathrm{~nm}$. With a galvanometric laser scanner (intelliScan10se, SCANLAB GmbH, Puchheim, Germany), areas of $5 \times 5 \mathrm{~mm}^{2}$ were processed by scanning the laser beam over the sample surface in parallel lines. The distance between two lines was adapted in order to generate homogeneous surface areas. By shifting the focus position automatically with a dynamic beam expander (VarioScan20i_de, SCANLAB GmbH, Puchheim, Germany) the beam diameter on the sample surface was modified. The laser beam was focused by a telecentric F-Theta lens (Sill Optics GmbH \& Co. KG, Wendelstein, Germany) with a focal length of $100 \mathrm{~mm}$. For selected experiments, the sample surface was purged with a gas (helium, argon or nitrogen) from a nozzle placed opposite to a suction system [see Fig. 2(a)].

The laser was operated at a pulse repetition rate of $f=1 \mathrm{MHz}$ and the average laser power was the same for all angles of incidence. The beam waist diameter was experimentally determined to amount $2 w_{0}=12 \mu \mathrm{m}$. By a defined defocusing, the beam diameter $2 w$ at the sample surface was set to about $2 w=25.3 \mu \mathrm{m}$. The average laser power was adjusted so that the peak fluence was $\phi_{0}=0.17 \mathrm{~J} / \mathrm{cm}^{2}$ at normal incidence. Because the effective beam cross section has an elliptic shape for an angle of incidence different from $0^{\circ}$, the peak fluence decreases in those experiments. Using a scan speed of $\mathrm{v}=1 \mathrm{~mm} / \mathrm{s}$ and a line offset of $\Delta y=6 \mu \mathrm{m}$, the effective number of pulses is $N_{\text {eff_1D }} \approx 2.53 \times 10^{4}$ and for $2 \mathrm{D}$ area scanning it amounts to $N_{\text {eff_2 }}=\pi w 0^{2} \times f /(\mathrm{v} \cdot \Delta \mathrm{y}) \approx 8.4 \times 10^{4}$ at normal incidence. For the different angles of incidence, these numbers change depending on the scan direction. For scanning perpendicular to the tilting axis [Fig. 2 (b), top], the distance between two lines was kept constant. Since the effective beam cross section has an elliptic shape at a tilted sample surface, $N_{\text {eff_1D }}$ and $N_{\text {eff_2D }}$ are larger in the scan direction compared to the perpendicular (non-tilted) case. For scanning parallel to the tilting axis direction [Fig. 2(b), bottom], the beam diameter in the scan direction 


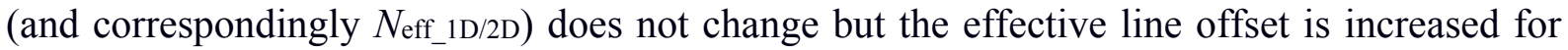
larger angles of incidence due to the projection of the line pattern on the sample surface.

At FORTH, 40-mm diameter samples were placed on a motorized 3-axis, linear translation stage. The fs-laser processing was performed at normal incidence with the sample surfaces placed close to the focal plane of a spherical bi-convex lens with a focal length of $50 \mathrm{~mm}$. At this position a Gaussian beam waist diameter of $2 w_{0}=140 \mu \mathrm{m}$ was measured by a CCD camera. In all cases an $\mathrm{Yb}: \mathrm{KGW}$ laser with central wavelength of $\lambda=1026 \mathrm{~nm}$, pulse duration $\tau=170 \mathrm{fs}$ and repetition rate $f=1 \mathrm{kHz}$ was used for irradiation.

In the case of laser processing at high repetition rates (CSIC), the implemented system was a Satsuma Yb-fiber laser from Amplitude Systems (Pessac, France), which delivers pulses of $\tau=500 \mathrm{fs}$ duration at a central wavelength of $\lambda=1030 \mathrm{~nm}$, with repetition rates $f$ up to $2 \mathrm{MHz}$ and a maximum pulse energy of $10 \mu \mathrm{J}$. The pulse energy was controlled by a rotating halfwave plate placed in front of a thin film polarizer. A second half-wave plate was used to define the polarization state of the pulses before reaching the sample. Focussing and scanning over the sample surface was performed by a computer controlled galvanometric scanner (max. speed: $12 \mathrm{~m} / \mathrm{s}$ ) coupled with an F-Theta lens with a focal length of $100 \mathrm{~mm}$ yielding a constant spot diameter $\left(1 / e^{2}\right.$ criterion) $2 w_{0}=39 \mu \mathrm{m}$ over an area of $1 \times 1 \mathrm{~cm}^{2}$.

After laser processing, all samples were cleaned either in acetone or isopropanol using an ultrasonic bath. Since both, acetone and isopropanol, are organic solvents with comparable polarity, we do not expect significant differences in the cleaning procedures performed at the different institutes. All laser processed surfaces were characterized by scanning electron microscopy (SEM) available at all institutes. On selected surface areas contact angle (CA) measurements and fluid transport tests were performed. The contact angle (wetting) measurements were performed at FORTH in a side-view geometry with a camera imaging a droplet parallel to the laser structured surface plane $\left(90^{\circ}\right)$ - compare the setup provided in reference [13]. This configuration allows to measure the static CA of the irradiated surfaces, using an OCA 35 system from DataPhysics Instruments GmbH (Filderstadt, Germany). A $4 \mu 1$ droplet of distilled water or commercial engine oil (Shell Rimula) was gently positioned on laser-treated areas using a microsyringe and droplet contour profiles were captured with the camera to measure the contact angle formed at the liquid-solid interface. For statistics, the (average) contact angle was determined as the mean value obtained from five subsequent measurements on each surface. After each consecutive measurement, water droplets remaining on the surface were removed by applying pressurized nitrogen gas, which completely dried the area of interest. For the gradient liquid motion, the sample was placed on a fixed mechanical base, levelled to the ground plane. A Sony RX100IV camera was used to record a video of the liquid movement on the laser processed sample areas in top view at 50 frames per second.

\section{RESULTS AND DISCUSSION}

\subsection{Identification of different morphologies on $42 \mathrm{CrMo} 4$ steel}

In order to systematically study the different types of laser-induced morphologies, areas of $2 \times 1 \mathrm{~mm}^{2}$ were laser processed using different sample scan velocities and laser pulse energies (BAM: $30 \mathrm{fs}, 790 \mathrm{~nm}, 1 \mathrm{kHz}$ ). SEM revealed some characteristic surface morphologies at the steel surfaces, such as laser-induced periodic surface structures (LIPSS) [16-20], grooves [21], and spikes (also named cones) [19, 21-24]. All of these structures are formed under homogeneous laser-irradiation in a "self-ordered" way with particular spatial periods and orientations to the laser polarization. Regarding the formation mechanisms of the different laser-induced morphologies, the reader is referred to literature $[16,18,20,24]$. In the following the LIPSS will be termed ripples for brevity. In general, on metals, two different types of ripples were identified in the literature: high-spatial-frequency LIPSS (HSFL) feature 
spatial periods $\Lambda$ between $100 \mathrm{~nm}$ and $200 \mathrm{~nm}$ and an orientation parallel to the laser beam polarization. Low-spatial-frequency LIPSS (LSFL) exhibit periods close to the laser wavelength $\lambda$ and an orientation perpendicular to the laser polarization [20].

By systematically varying the effective number of laser pulses $\left(N_{\text {eff_1D }}\right)$ from 5 up to 1200 along with peak laser fluences $\left(\phi_{0}\right)$ ranging from 0.15 to $2.5 \mathrm{~J} / \mathrm{cm}^{2}, 82$ different areas were fslaser processed. By a careful analysis of those laser-processed surfaces using SEM, four characteristic surface morphologies, i.e. HSFL, LSFL, grooves and spikes, could be identified on the $42 \mathrm{CrMo} 4$ steel. With those results in hand, a morphological map for area processing [Fig. 3 (a)] was constructed for that particular alloyed steel material. This graph reveals that HSFL-ripples are solely formed for a very low number of laser pulses $\left(N_{\text {eff_1D }}<100\right)$ and at maximum peak fluences of $\phi_{0} \sim 0.25 \mathrm{~J} / \mathrm{cm}^{2}$, close to the damage threshold of the material. LSFL-ripples are formed either at $N_{\text {eff_1D }} \leq 20-40$ and for fluences up to a few $\mathrm{J} / \mathrm{cm}^{2}$, or at $N_{\text {eff_1D }}$ up to 100 and for peak fluences of $0.25 \mathrm{~J} / \mathrm{cm}^{2}$. Spikes are formed at high fluences and number of pulses $\left(N_{\text {eff_1D }} \geq 200, \phi_{0} \geq 1.5 \mathrm{~J} / \mathrm{cm}^{2}\right)$. The grooves appear as a transitory morphology between the LSFL-ripples and the spikes. Transition areas between the different morphologies, where LSFL/grooves or grooves/spikes mixtures are present, could be observed.

Examples for top-view SEM micrographs of the different characteristic surface morphologies are given in Fig. 3. Ripples with periods $\Lambda$ of $550 \pm 80 \mathrm{~nm}$ (LSFL) are depicted in Fig. 3(b), while grooves with distances of $\sim 3.2 \mu \mathrm{m}$ and spikes with typical lateral extents between 6 and $13 \mu \mathrm{m}$ are shown in Fig. 3(c) and 3(d). Additionally, Fig. 3(d) (bottom) features a tilted-view SEM micrograph of the spikes structures on $42 \mathrm{CrMo} 4$, highlighting the similarity to the surface structures found on bark bugs [compare Fig. 1(c)]. The dimensions of the spikes were determined by white light interference microscopy (WLIM), resulting in the following values: height $\sim 9 \mu \mathrm{m}$, length $\sim 10 \mu \mathrm{m}$, width $\sim 6.5 \mu \mathrm{m}$ and an average distance between the spikes of $\sim 9 \mu \mathrm{m}$ (data not shown here). This yields the aspect ratios of length-to-width: $1.7: 1$, lengthto-height: $1.1: 1$, and width-to-height: $0.7: 1$ Whereas the lateral dimensions of the spikes are comparable to those found on the bark bugs, the height of the spikes is about 2.5 times larger than that of the bark bugs - compare introduction.

The spatial period of laser-induced nano- and microstructures depends on the processing parameters, such as wavelength, peak fluence, number of pulses per spot and, to a minor extent also on the pulse duration [20]. While ripples and grooves both clearly depend on the laser beam polarization (LSFL: perpendicular to polarization, HSFL and grooves: parallel to polarization), the spikes morphology is non-directional. However, the latter exhibits a clear correlation with the local laser fluence. Larger distances are observed between neighboured spikes in regions where the Gaussian-like fluence distribution maximizes (perpendicular to the line scanning direction) [13]. Moreover, the spikes formed here upon fs-laser scan processing represent a multi-scale structure, where the micrometre-sized spike-like features are additionally covered by a nanoscale roughness (including residuals of LSFL-ripples).

The map shown in Fig. 3(a) is based on results obtained at BAM $[\lambda=790 \mathrm{~nm}, \tau=30 \mathrm{fs}$, $f=1 \mathrm{kHz}]$. Analog maps were generated at each institute and may vary somewhat, due to different laser parameters $(\lambda, \tau, f)$. Laser-induced spikes could be achieved at different parameters: at BAM, fluences $\geq 1.5 \mathrm{~J} / \mathrm{cm}^{2}$ and moderate $N_{\text {eff_1D }}(200-1200)$ were used, whereas at FORTH $[\lambda=1026 \mathrm{~nm}, \tau=170 \mathrm{fs}, f=1 \mathrm{kHz}]$ somewhat lower fluences $\left(\sim 1 \mathrm{~J} / \mathrm{cm}^{2}\right)$

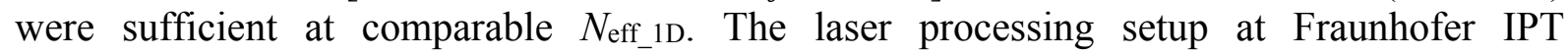
$[\lambda=532 \mathrm{~nm}, \tau=9 \mathrm{ps}, f=1000 \mathrm{kHz}]$ is operated at three orders of magnitude larger pulse repetition rate, resulting in much higher $N_{\text {eff_1D }}$ (in the range of $10^{4}$ ). Hence, significantly lower fluences $\left(<0.2 \mathrm{~J} / \mathrm{cm}^{2}\right)$ are required to generate spikes.

\subsection{Different approaches for optimum similarity to bark bug structures}


In section 3.1 it has been demonstrated that the spike like structures found underneath the wings of bark bugs can be processed on steel surfaces by irradiation with ultrashort laser pulses. For mimicking the natural appearance of the bark bug structures more precisely, namely the tilting of the spikes, compare Fig. 1 (c), different approaches were followed, which are described in the following two sub-sections. This includes laser processing of tilted sample surfaces (section 3.2.1) and the additional variation of the surrounding atmosphere (section 3.2.2). Since tilting of the sample and purging the sample surface during laser irradiation is possible without further modification of the setup at Fraunhofer IPT, see section 2 and Fig. 2, the results presented below (3.2.1 and 3.2.2) were obtained using this laser processing setup $[\lambda=532 \mathrm{~nm}, \tau=9 \mathrm{ps}, f=1000 \mathrm{kHz}]$.

\subsubsection{Effect of the irradiation angle}

Spikes pointing normal to the surface have been fabricated using a ps-laser $[\lambda=532 \mathrm{~nm}, \tau=9$ ps and $f=1000 \mathrm{kHz}]$ at normal incidence of the laser beam on the sample surface $\left(0^{\circ}\right.$ angle of incidence). Furthermore, experiments with tilted samples (angle of incidence $20^{\circ}$ and $40^{\circ}$ ) have been carried out by ps-laser processing to produce spikes with a preferred direction. In Fig. 4, the top-view SEM micrographs of the fabricated spikes for a scanning direction perpendicular [(d) - (f)] and parallel [(a) - (c)] to the tilting axis are shown. It is clearly visible that the spikes exhibit a directionality for tilted irradiation. This effect increases with increasing angle. The spikes for the two different scan strategies differ significantly. A reason for this might be the fact that $N_{\text {eff_1D/2D }}$ differs for the two scan directions (see the experimental section 2). For scanning parallel to the tilting axis, $N_{\text {eff_1D/2D }}$ is kept constant, whereas when scanning perpendicular to the tilting axis, it is higher. Moreover, the local fluence decreases with increasing angle of incidence, as the average power was kept constant in the experiments. The tilted spikes laser processed at an angle of incidence of $40^{\circ}$ with line scans perpendicular to the tilting axis mimic the natural model best - compare Fig. 1(c). Note that, in contrast to the fs-laser processed spikes described in section 3.1, here $N_{\text {eff } 1 \text { D }}$ is significantly higher $\left(2.5-3.5 \times 10^{4}\right.$ in contrast to 400). As a consequence, the LIPSS substructure on the spike is not observed [25].

\subsubsection{Effect of the irradiation atmosphere}

In order to achieve the optimum similarity to the biological prototype, i.e., the tilted spikes found on the integument of bark bugs, an additional approach was followed by varying the irradiation atmosphere. As previously reported for fs-laser spot-processing of silicon, the atmosphere may influence the appearance (sharpness) [26] and wettability [27, 28] of the spike structures. For this purpose, the sample surface was purged with either nitrogen, argon or helium. In Fig. 5, corresponding SEM micrographs for ps-laser irradiation at angles of $0^{\circ}$ and $40^{\circ}$ and scanning direction parallel to the tilting axis are shown. Those results reveal that the use of inert gases does not result in the intended enhanced sharpness of the spikes. The variations for example in the size of the spike structures and the change of the substructures are rather attributed to small changes in the average laser power along with the inherent irregularity in the spike formation process. The differences observed here in contrast to Ref. [26] regarding the sensitivity of spikes to the atmosphere may lie in the scan-processing approach realized in this work and the different materials and laser processing parameters. Measurements of the static CA on the spike structures shown in Fig. 4 and 5 revealed no significant differences between the samples processed in different atmospheres and at different irradiation angles $\left(\mathrm{CA} \sim 145^{\circ} \pm 8^{\circ}\right)$.

The results presented in section 3.2 demonstrate the processing of titled spike structures on steel, successfully mimicking the micro-ornamentation found on bark bugs D. magnus [see Fig. 1(c)].

\subsection{Fluid transport}


Additional to the shape, also the functionality, namely directional fluid transport, of the bark bug structures are desired to be mimicked. To this end, spatial gradients featuring different surface morphologies ranging from ripples to spikes were fs-laser processed and tested using water (section 3.3.1) and engine oil (section 3.3.2). Additionally, the tilted spike structures described in section 3.2.1 were also tested with oil.

\subsubsection{Water transport}

At FORTH, gradient structures were produced upon processing an $8 \times 15 \mathrm{~mm}^{2}$ area by progressively attenuating the laser beam fluence that irradiates the target, while keeping the $N_{\text {eff_1D }}$ constant. Accordingly, in the beginning, where the fluence is at its maximum $\left(\phi_{0}=1.05 \mathrm{~J} / \mathrm{cm}^{2}\right)$, we observe the formation of spikes at the left area of the irradiated surface [see Fig. 6(a)]. Upon reducing the fluence, we observe the formation of grooves [Fig. 6(b)] in the central region of the surface. Finally, when the fluence is at its minimum $\left(\phi_{0}=0.07 \mathrm{~J} / \mathrm{cm}^{2}\right)$ we observe the formation of LSFL-ripples at the right area [Fig. 6(d)].

In accordance with the literature [29-31] the laser processed steel surfaces were found to be superhydrophilic (i.e. $\mathrm{CA}<50^{\circ}$ ) for several days right after the processing, regardless the type of the structures attained. The initial superhydrophilicity is attributed to the laser-induced surface oxidation, which occurs during irradiation in air environment [29] and the removal of water molecules [32]. It was reported in literature that the CA progressively increases to a terminal value after 9-10 days of storage in ambient air [29, 31, 32]. The change of the wetting behaviour can be explained by changes of the surface chemistry [31, 33, 34], which includes the adsorption of hydrocarbon molecules from the atmosphere on the irradiated surface [29] and a change of surface polarity [32].

The fluid transport properties of the gradient structures were measured 1 day after irradiation, by gently applying a $\sim 8 \mu 1$ droplet of deionized water in the middle of the treated surface. The liquid droplet swiftly spread at a velocity estimated to be $\sim 75 \mathrm{~mm} / \mathrm{s}$, completely covering the upper area of the structured surface (see Fig. 7), where the fluence during irradiation was at its maximum, i.e., where the micrometre-sized spike structures were generated. Liquid motion to the lower part of the surface was not observed. When placing the droplet onto the LSFL covered area, water droplets swiftly spread to the opposite end, eventually covering the whole surface.

The experimental observations can be interpreted in the framework of a simplified model accounting for the equilibrium of the different types of forces governing the droplet motion along a surface tension gradient, as described in reference [35].

$$
\sum F^{\prime}=F_{a o t}^{\prime}-F_{b y s}-F_{\text {wos }}^{\prime}=m a
$$

According to equation (1), the total force acting on the droplet, is comprised of different contributions: the actuation force, $F_{\text {act }}$, moves the droplet across the surface and originates from the variable surface energy and, in turn, wettability of the liquid-solid interface. The counteracting resistance force comprises two components: the hysteresis force, $F_{\text {hys, }}$, resulting from the hysteresis phenomenon occurring before a droplet begins to move, and the viscous force, $F_{\text {vis, }}$ of the liquid, acting during the droplet motion. Assuming that $F_{\text {hys }}$ is equal in both parts of the surface, the surface tension gradient is higher in the spikes covered area, since the micrometre-sized spike structures are more hydrophilic than the LSFL (CA of $\sim 0^{\circ}$ compared to $\sim 40^{\circ}$, respectively). This is reasonable according to the Wenzel model $\cos \theta_{\mathrm{w}}=\mathrm{r} \times \cos \theta_{0}$ [36], where $\theta_{\mathrm{w}}$ is the apparent contact angle, $r$ is the roughness ratio, which describes the ratio of true area of the solid surface to the apparent area underneath the droplet. $\theta_{0}$ is the contact angle on a flat surface of the same nature as the rough one. Since $r$ is higher for spikes than for LSFL [27] it is concluded that the spikes area is more hydrophilic that the LSFL area, which is in line with the measured contact angles. 
The same experiment was repeated 14 days after irradiation to test for possible water transport after storing the sample in ambient conditions. In this case no fluid motion was observed, attributed to the increase in hydrophobicity, which was more pronounced in the spiked regions (Fig. 6 (a)), exhibiting higher hydrophobicity compared to the rest of the areas. Nevertheless, no directional liquid transport was observed. This can be seen in Fig. 14, which depicts water droplets on the gradient surface taken $\sim 1$ minute after placing the droplets of water on three different regions of the gradient surface. These measurements were repeated 28 days after irradiation. As the degree of hydrophobicity of the structured surfaces was further enhanced, no water spreading could be observed either. In particular, the droplet sticks on the surface, possibly due to high hysteresis forces, and cannot move from more to less hydrophobic areas. This evolution of wetting behaviour of water is attributed to changes in surface chemistry, which are enhanced by the surface structure [29].

\subsubsection{Oil transport}

Due to the fact that laser processed surfaces become hydrophobic and in turn oleophilic several days after irradiation [29, 30], the samples irradiated at Fraunhofer IPT and BAM could only be tested with respect to oil transport (at FORTH). This approach is in line with the function executed by the spike structures on the bark bug's integument - see introduction. On the ps-laser processed tilted spike structures [compare Fig. 4(f)] an oil droplet placed in the centre spreads omnidirectionally, as can be seen in Fig. 8. This result corroborates the findings by Plamadeala et. al. [11], who mimicked bark bug structures in polymers by direct laser writing. Only when covering the pointy microstructures with a coverslip - similar to the tilted spikes on the bark bugs, which are covered by their wings - they observed unidirectional spreading of an applied oil droplet in the opposite direction of the microstructures' tips.

At BAM, surface areas of $5 \times 15 \mathrm{~mm}^{2}$ featuring a size gradient of micro- and nanostructures were fs-laser processed $\left(\lambda=790 \mathrm{~nm}, \tau=30 \mathrm{fs}, f=1 \mathrm{kHz} ; N_{\text {eff_1D }}=5-1200 ; \phi_{0}=2.0,2.5\right.$ and $3.0 \mathrm{~J} / \mathrm{cm}^{2} ; \Delta y=0.04 \mathrm{~mm}$; see Fig. 9) on $42 \mathrm{CrMo} 4$ steel by varying $N_{\text {eff_1D. During meandering }}$ scanning of the surface using fs-laser pulses, the scan speed was gradually increased (every 5 lines along the $x$-axis) from 0.093 to $22 \mathrm{~mm} / \mathrm{s}$, resulting in $N_{\text {eff_1D }}$ ranging from 1200 at the top to $\sim 5$ at the bottom of the area. This procedure was repeated for three different peak fluences $\phi_{0}=2.0,2.5$ and $3.0 \mathrm{~J} / \mathrm{cm}^{2}$, generating three areas on one sample [Fig. 9(a) and (b)]. A first hint on the successful generation of different morphologies was already given by inspection with the bare eye. The fs-laser processed areas are black at the top and become gradually lighter towards the bottom [Fig. 9(a)]. When changing the angle of inspection, a blue-green coloration at the bottom of the areas can be discerned [Fig. 9 (b)], which arises from LSFL-ripples that act as diffractive gratings. [20, 37] When further investigating the gradient structures using SEM, spikes could be identified at the top [Fig. 9(c)], grooves in the centre [Fig. 9(d)] and LSFL at the bottom [Fig. 9(e)] of all three areas. Accordingly, a size gradient from micrometre-sized spikes down to nanometre-sized ripples was successfully generated. Comparing the three areas laser processed at different peak fluences, the main distinction is that at lower $\phi_{0}$ the transition from spikes to grooves and ripples starts earlier, which is also related to the colour of the areas [compare Fig. 9(a) and (b)].

Approximately two weeks after fs-laser processing of the described gradient structures, contact angles (CAs) of water were measured on the surfaces at FORTH. The results are listed in Tab. 2 and side view photographs of selected CA measurements are shown in Fig. 10. From those results it can be deduced that in the top region spikes with CAs of $\sim 134-137^{\circ}$ were generated. The grooves covered central region features CAs of $139-140^{\circ}$ at $\phi_{0}=3.0$ and $2.5 \mathrm{~J} / \mathrm{cm}^{2}$. In case of $\phi_{0}=2.0 \mathrm{~J} / \mathrm{cm}^{2}$ the CA in the central region is significantly lower, i.e. $122^{\circ}$, which points to the presence of ripples. At the ripples covered bottom region CAs of $109-122^{\circ}$ were measured. Consequently, the whole area of gradient structures, independent

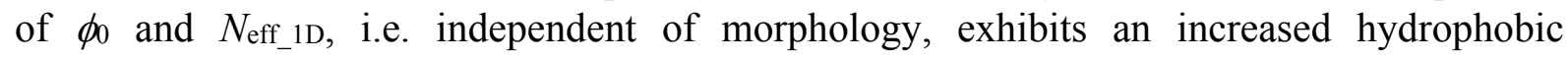


behaviour hindering water spreading two weeks after the laser processing. Hence, wetting properties of engine oil (Shell Rimula) were investigated on the laser irradiated surfaces. The groove and spike areas exhibited superoleophilic behaviour (i.e. $\mathrm{CA}=0^{\circ}$ ), while the LSFL area showed a CA of $38.5 \pm 3^{\circ}$. Furthermore, the CA measurements were repeated 14 and 28 days after irradiation, to further investigate possible alterations of the wetting properties as a function of time. It was observed that all the surfaces preserved their high oleophilicity regardless of the time the measurement was carried out.

Thus, engine oil (Shell Rimula) was also used for fluid transport measurements on the gradient structures. When applying a droplet of $8 \mu 1$ of the oil on the grooves-covered centre of the fs-laser processed area it spreads unidirectionally towards the larger spikes structures (top) [see Fig. 11]. For the area processed at $\phi_{0}=3.0 \mathrm{~J} / \mathrm{cm}^{2}$ a velocity of $\sim 0.04 \mathrm{~mm} / \mathrm{s}$ was determined. On the areas processed at lower fluences the oil spread slightly slower with $0.03 \mathrm{~mm} / \mathrm{s}$.

Finally, also the gradient structures featuring a fluence gradient (compare section 3.3.1) were tested regarding oil transport about one month after they have been fs-laser processed at FORTH. An oil droplet placed in the centre of the gradient-processed $8 \times 15 \mathrm{~mm}^{2}$ surface area moved unidirectionally towards the larger spike structures, as seen for the $N_{\text {eff-gradient }}$ structures, at a velocity of $0.05 \mathrm{~mm} / \mathrm{s}$. This is due to the fact that the surface tension gradient is higher in the area of more the oleophilic spike structures $\left(\mathrm{CA}=0^{\circ}\right)$ compared to the LSFL $\left(\mathrm{CA}=38.5 \pm 3^{\circ}\right)$.

\subsection{Area upscaling}

Microfabrication with modern high repetition rate lasers offers a clear advantage over the more traditional low repetition rate lasers: the possibility to speed up the overall fabrication process in order to meet industrial requirements. To name one example, it has been demonstrated that the use of such lasers allows the rapid fabrication of high quality diffraction gratings based on LSFL-ripples in chromium [38]. Yet, no comparative study was performed, investigating the influence of the repetition rate on the final structures produced.

In the present case of $42 \mathrm{CrMo} 4$ steel, it was investigated in detail if at such high repetition rates heat accumulation effects may induce changes in the structure formation or even lead to changes in the wetting properties. For this purpose, we have performed laser processing of extended areas with different repetition rates, keeping the two key parameters $\phi_{0}$ and $N_{\text {eff_1D }}$ constant.

Fig. 12 shows SEM micrographs of the surface morphology for different repetition rates at constant peak fluence $\left(2.0 \mathrm{~J} / \mathrm{cm}^{2}\right)$ and $N_{\text {eff_1D }}$ (except at $1 \mathrm{MHz}$ where this high fluence could not be reached). Consequently, the scanning speed in each micrograph was adjusted to keep a

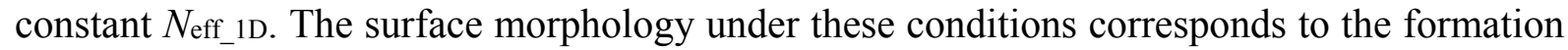
of spike-like structures. It can be seen that at low-to-moderate repetition rates [Fig. 12(a) (d)], the size, homogeneity and orientation of the structures is essentially the same. When increased up to $500 \mathrm{kHz}$ [Fig. 12(e)] a marked change in the appearance of the structures, especially in the inter-spike distance, can be noted, but the overall spike structure remains intact. It is important to note that these repetition rate thresholds depend on the number of effective pulses that impinge the sample, the repetition rate, and the energy of the laser pulse, which are specific of the implemented experimental setup. At $f=1 \mathrm{MHz}$ or higher, the spike structure disappeared and strong signs of surface melting caused by the massive heat accumulation could be observed, leaving behind a randomly porous structure. From an application point of view it can be concluded that under the present conditions $500 \mathrm{kHz}$ is the highest repetition rate possible for spike generation implementing the current laser processing setup $\left(2 w_{0}=39 \mu \mathrm{m}, \phi_{0}=2 \mathrm{~J} / \mathrm{cm}^{2}, \Delta y=30 \mu \mathrm{m}, \mathrm{v}_{\mathrm{x}}=50 \mathrm{~mm} / \mathrm{s}\right)$. These specific conditions translate to a processing time of $1 \mathrm{~min} / \mathrm{cm}^{2}$. Indeed, the overall processing time could be 
decreased by using a larger laser spot, keeping the laser fluence and the effective number of pulses $N_{\text {eff_1D }}$ constant and increasing $\Delta y$ accordingly.

The evolution of the contact angle of a water droplet placed on the areas shown in Fig. 12 containing spike structures as a function of the different repetition rates is shown in Fig. 13. It can be seen that the influence of the repetition rates on the contact angle is rather low, keeping a virtually constant value of $136^{\circ}$ (except for the value at $10 \mathrm{kHz}$ which features a large error bar, since only a single CA measurement could be performed. This particular value has to be taken with care, since no sharp reflection was observed on the sample surface.). This result is remarkable since it shows that upscaling with high repetition rate lasers is feasible, keeping the wettability properties unchanged.

\section{CONCLUSIONS}

By systematically varying parameters such as peak fluence, number of effective pulses per spot, irradiation angle and atmosphere during laser processing using ultrashort pulses (fs-ps) the micro-ornamentation found on bark bugs was successfully mimicked on steel. The shape of the micrometre-sized tilted spikes found underneath the wings of the bark bug D. magnus (see Fig. 1) was reproduced on alloyed $42 \mathrm{CrMo} 4$ steel when irradiating the sample under an angle of $40^{\circ}$ in air at moderate fluences $\left(\phi_{0}=0.13 \mathrm{~J} / \mathrm{cm}^{2}\right)$ and high $N_{\text {eff_1D }}\left(\approx 3.3 \times 10^{4}\right)$ with pslaser pulses. Furthermore, the hydrophobic functionality of the natural prototype could be achieved on the laser processed steel surfaces. Oil transport on those surfaces was found to be omnidirectional. On fs-laser processed areas featuring size gradients from micrometre-sized spikes to nanometre-sized ripples the unidirectional fluid transport identified on the bark bugs surface was effectively imitated for water (immediately after the laser processing) as well as for engine oil. The fastest unidirectional fluid transport was observed for water featuring velocities up to $75 \mathrm{~mm} / \mathrm{s}$, while for engine oil the transport was significantly slower in the range of $0.03-0.05 \mathrm{~mm} / \mathrm{s}$. In order to make this process attractive for technical applications, laser processing of those functional microstructures was tested at higher repetition rates, proving that the formation of spike structures is feasible at repetition rates up to $500 \mathrm{kHz}$ without losing its surface wetting properties.

\section{ACKNOWLEDGEMENTS}

The Authors would like to thank S. Benemann, (BAM 6.1) for the SEM characterizations, A. Hertwig (BAM 6.7) for WLIM measurements, and S. Binkowski (BAM 6.3) for polishing the steel samples. This work has received funding from the Horizon 2020 European Union's research and innovation programme under grant agreement No. 665337 ("LiNaBioFluid"; URL: www.laserbiofluid.eu). 


\section{REFERENCES}

1. K. Liu, X. Yao, L. Jiang, Chem. Soc. Rev. 39, 3240-3255 (2010).

2. $\quad$ R. Silberglied, A. Aiello, Science 207, 773-775 (1980).

3. E. F. Contreras, M. D. Coscaron, Zootaxa 3500, 1-35 (2012).

4. E. Heiss, Anales Inst. Biol. Univ. Nac. Auton. Mexico Ser. Zool. 61, 279-296 (1990).

5. T. van der Heyden, J. M. Díaz, Arquivos Entomolóxicos 15, 371-373 (2016).

6. D. Chandra, S. Yang, Langmuir 27, 13401-13405 (2011).

7. M. C. Larivière, A. Larochelle, Kataloge der OÖ. Landesmuseen Neue Serie 50, 181214 (2006).

8. B. S. Krall, R. J. Bartelt, C. J. Lewis, D. W. Whitman, J. Chem. Ecol. 25, 2477-2494 (1999).

9. J. R. Aldrich, Annu. Rev. Emntomol. 33, 211-238 (1988).

10. J. Raška, PhD Thesis, Charles University Prague, 2009.

11. C. Plamadeala, F. Hischen, R. Friesenecker, R. Wollhofen, J. Jacak, G. Buchberger, E. Heiss, T. A. Klar, W. Baumgartner, J. Heitz, Roy. Soc. Open. Sci. 4, 160849 (2017).

12. P. Kment, J. Vilimova, Zootaxa 2706, 1-77 (2010).

13. U. Hermens, S. V. Kirner, C. Emonts, P. Comanns, E. Skoulas, A. Mimidis, H. Mescheder, K. Winands, J. Krüger, E. Stratakis, J. Bonse, Appl. Surf. Sci. 418, 499-507 (2017).

14. J. M. Liu, Opt. Lett. 7, 196-198 (1982).

15. J. Bonse, G. Mann, J. Krüger, M. Marcinkowski, M. Eberstein, Thin Solid Films 542, 420-425 (2013).

16. J. E. Sipe, J. F. Young, J. S. Preston, H. M. van Driel, Phys. Rev. B 27, 1141-1154 (1983).

17. J. Bonse, J. Krüger, S. Höhm, A. Rosenfeld, J. Laser Appl. 24, 042006 (2012).

18. A. Y. Vorobyev, C. Guo, Laser \& Photonics Reviews 7, 385-407 (2013).

19. G. D. Tsibidis, C. Fotakis, E. Stratakis, Phys. Rev. B 92, 041405 (2015).

20. J. Bonse, S. Höhm, S. V. Kirner, A. Rosenfeld, J. Krüger, IEEE J. Sel. Top. Quant. 23, 9000615 (2017).

21. K. Ahmmed, C. Grambow, A.-M. Kietzig, Micromachines 5, 1219 (2014).

22. J. Bonse, S. Baudach, J. Krüger, W. Kautek, M. Lenzner, Appl. Phys. A 74, 19-25 (2002).

23. T. Yong Hwang, C. Guo, J. Appl. Phys. 111, 083518 (2012).

24. E. J. Y. Ling, J. Saïd, N. Brodusch, R. Gauvin, P. Servio, A.-M. Kietzig, Appl. Surf. Sci. 353, 512-521 (2015).

25. K. Lange, M. Schulz-Ruhtenberg, J. Caro, ChemElectroChem 4, $570-576$ (2017).

26. T.-H. Her, R. J. Finlay, C. Wu, S. Deliwala, E. Mazur, Appl. Phys. Lett. 73, 16731675 (1998).

27. V. Zorba, E. Stratakis, M. Barberoglou, E. Spanakis, P. Tzanetakis, C. Fotakis, App. Phys. A 93, 819-825 (2008).

28. V. Zorba, E. Stratakis, M. Barberoglou, E. Spanakis, P. Tzanetakis, S. H. Anastasiadis, C. Fotakis, Adv. Mater. 20, 4049-4054 (2008).

29. A.-M. Kietzig, S. G. Hatzikiriakos, P. Englezos, Langmuir 25, 4821-4827 (2009).

30. A.-M. Kietzig, M. Negar Mirvakili, S. Kamal, P. Englezos, S. G. Hatzikiriakos, J. Adhes. Sci. Technol. 25, 2789-2809 (2011).

31. M. Martínez-Calderon, A. Rodríguez, A. Dias-Ponte, M. C. Morant-Miñana, M. Gómez-Aranzadi, S. M. Olaizola, Appl. Surf. Sci. 374, 81-89 (2016).

32. P. Bizi-bandoki, S. Valette, E. Audouard, S. Benayoun, Appl. Surf. Sci. 273, 399-407 (2013).

33. M. V. Rukosuyev, J. Lee, S. J. Cho, G. Lim, M. B. G. Jun, Appl. Surf. Sci. 313, 411417 (2014). 
34. J. Yong, F. Chen, Q. Yang, X. Hou, Soft Matter 11, 8897-8906 (2015).

35. I. Paradisanos, C. Fotakis, S. H. Anastasiadis, E. Stratakis, Appl. Phys. Lett. 107, 111603 (2015).

36. R. N. Wenzel, Ind. Eng. Chem. 28, 988-994 (1936).

37. A. Y. Vorobyev, C. Guo, Appl. Phys. Lett. 92, 041914 (2008).

38. A. Ruiz de la Cruz, R. Lahoz, J. Siegel, G. F. de la Fuente, J. Solis, Opt. Lett. 39, 2491-2494 (2014). 


\section{Tables}

\begin{tabular}{|c|c|c|c|c|}
\hline $\begin{array}{c}\text { Laser processing } \\
\text { parameters }\end{array}$ & BAM & IPT & FORTH & CSIC \\
\hline Wavelength $\lambda[\mathrm{nm}]$ & 790 & 532 & 1026 & 1030 \\
\hline Pulse duration $\tau[\mathrm{ps}]$ & 0.03 & 9 & 0.17 & 0.5 \\
\hline Repetition rate $f[\mathrm{kHz}]$ & 1 & 1000 & 1 & $10-1000$ \\
\hline Focus diameter $2 w_{0}[\mu \mathrm{m}]$ & $130 / 112$ & 12 & $130-140$ & 39 \\
\hline Line separation $\Delta y[\mu \mathrm{m}]$ & $40 / 50 / 100$ & 6 & 30 & 30 \\
\hline Scan velocity $\mathrm{v}_{\mathrm{x}}[\mathrm{mm} / \mathrm{s}]$ & $0.093-26$ & 1 & 0.4 & $1-200$ \\
\hline Angle of incidence $\left[{ }^{\circ}\right]$ & 0 & $0,20,40$ & 0 & 0 \\
\hline
\end{tabular}

Tab. 1 Parameters used for laser processing at the different institutes (BAM, Fraunhofer IPT, FORTH, CSIC).

\begin{tabular}{|c|c|c|c|}
\hline $\begin{array}{l}\text { Region } \\
\text { Morphology }\end{array}$ & $3.0 \mathrm{~J} / \mathbf{c m}^{2}$ & $\mathbf{2 . 5} \mathbf{~ J / \mathbf { c m } ^ { 2 }}$ & $\mathbf{2 . 0} \mathbf{~ J / \mathbf { c m } ^ { 2 }}$ \\
\hline Top / Spikes & $135^{\circ} \pm 4^{\circ}$ & $137^{\circ} \pm 5^{\circ}$ & $134^{\circ} \pm 5^{\circ}$ \\
\hline Centre / Grooves & $139^{\circ} \pm 5^{\circ}$ & $140^{\circ} \pm 5^{\circ}$ & $122^{\circ} \pm 4^{\circ}$ \\
\hline $\begin{array}{c}\text { Bottom / LSFL- } \\
\text { ripples }\end{array}$ & $122^{\circ} \pm 5^{\circ}$ & $119^{\circ} \pm 5^{\circ}$ & $109^{\circ} \pm 4^{\circ}$ \\
\hline
\end{tabular}

Tab. 2. Contact angles (CAs in ${ }^{\circ}$ ) of $4 \mu 1$ deionized water on fs-laser processed [Processing conditions: $\lambda=790 \mathrm{~nm}, \tau=30 \mathrm{fs}, f=1 \mathrm{kHz}]$ gradient structures on $42 \mathrm{CrMo} 4$ steel in dependence on the region/morphology within the laser processed area and peak fluence $\phi_{0}$. 


\section{Figures}

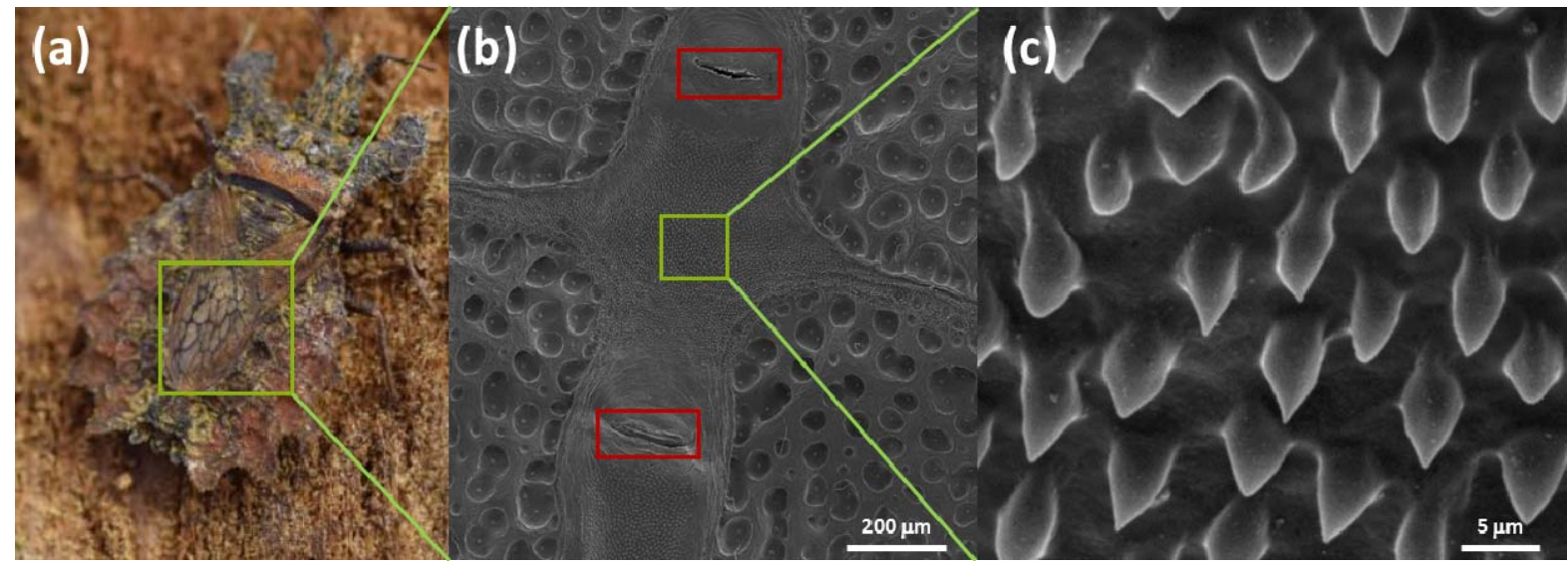

Fig. 1. (a) Optical image of Dysodius magnus camouflaging on bark (b) SEM micrograph of $D$. magnus' cuticle under the wing with the scent liquid-secreting gland openings (marked by red rectangles) and the complex surface micro-ornamentation. (c) A higher magnification of the micro-ornamentation region, where tilted spike-like microstructures can be seen in detail.
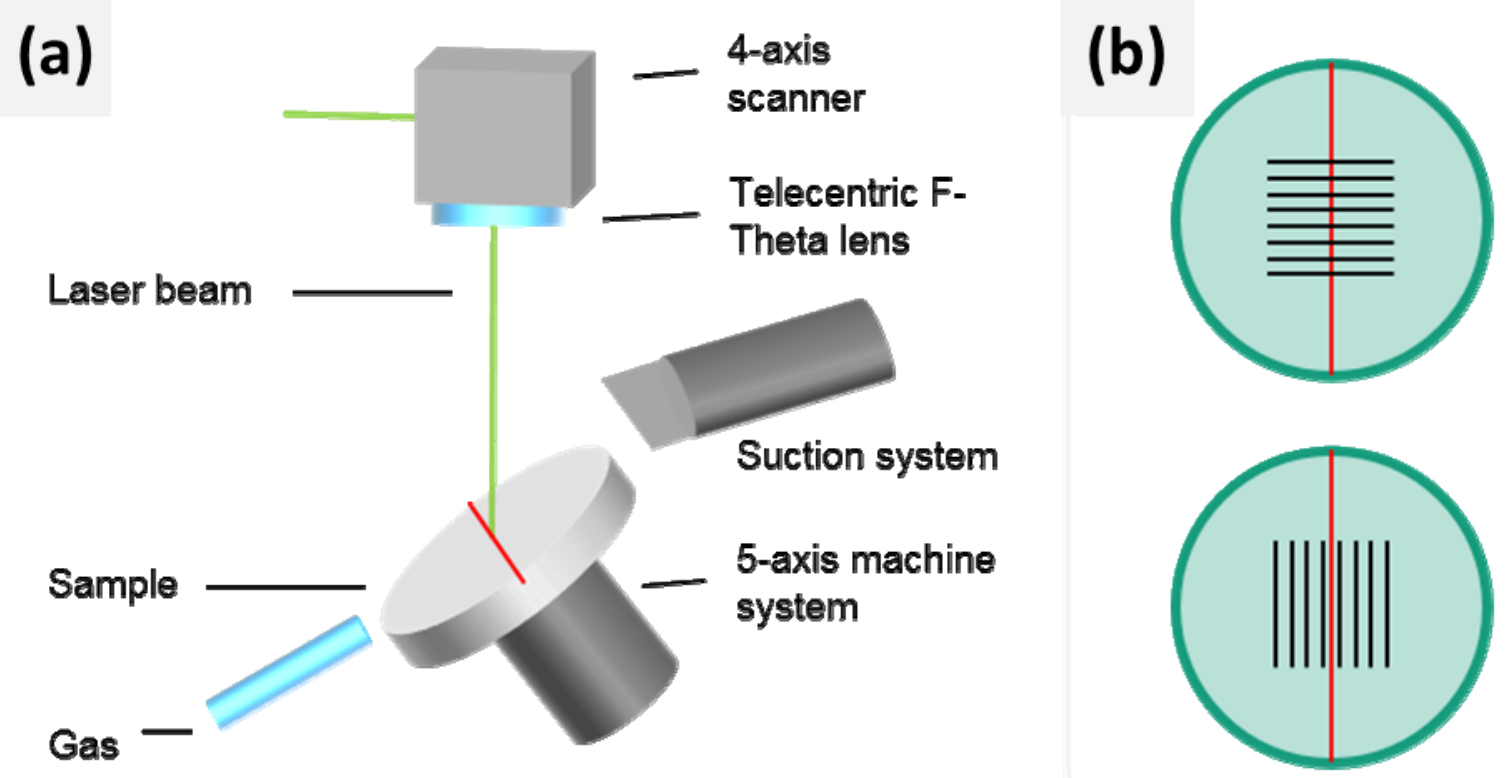

Fig. 2. (a): Setup for the laser structuring at the Fraunhofer IPT, (b): Schematic illustration of the laser scanning direction (black lines) with respect to the tilting axis of the sample surface (red line). 

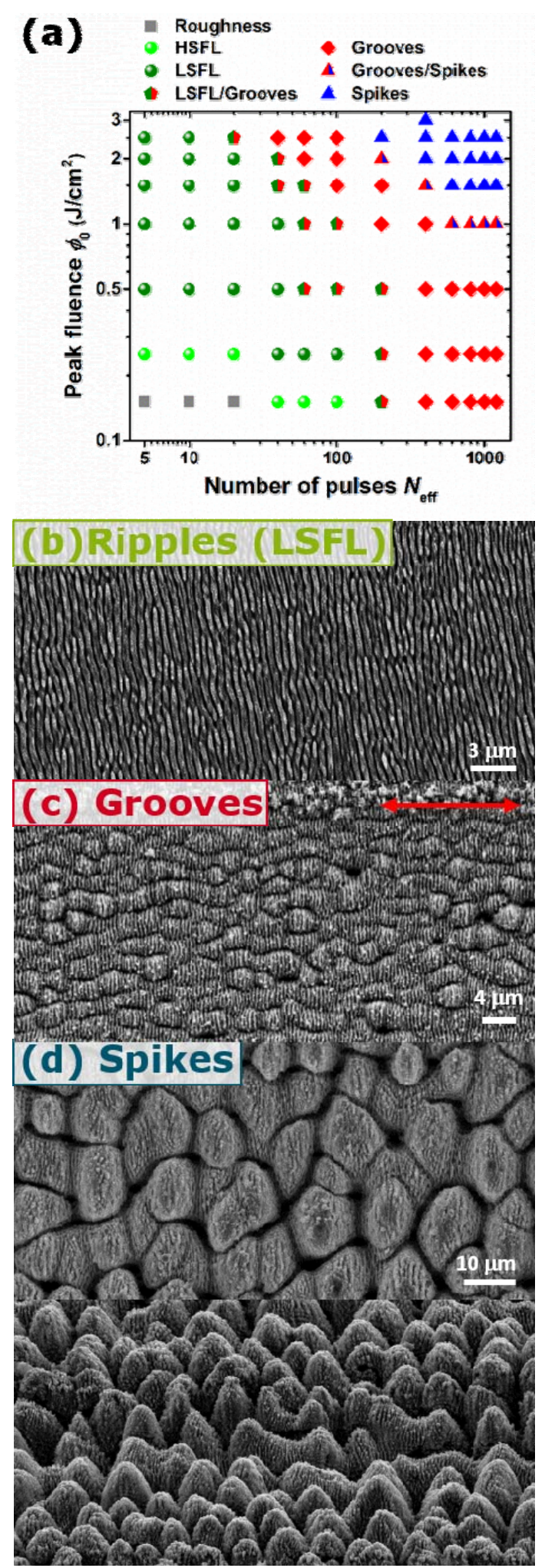

Fig. 3. (a) Morphological map of characteristic surface morphologies [ripples (HSFL and LSFL), grooves, spikes] formed on $42 \mathrm{CrMo} 4$ steel surfaces upon fs-laser area scanning at different irradiation parameters [Processing conditions: $\lambda=790 \mathrm{~nm}, \tau=30 \mathrm{fs}, f=1 \mathrm{kHz}$ ] Note the doublelogarithmical data representation. Corresponding top-view SEM micrographs of selected scanning fs-laser processed surface areas on steel covered by LSFL-ripples (b), grooves (c), and spikes (d; $0^{\circ}$ top-view and $60^{\circ}$ tilted view). Processing conditions: $\Delta y=50 \mu \mathrm{m}$; LSFL-ripples (b): $\phi_{0}=0.5 \mathrm{~J} / \mathrm{cm}^{2}, N_{\text {eff_1D }}=40$; grooves (c): $\phi_{0}=0.5 \mathrm{~J} / \mathrm{cm}^{2}, N_{\text {eff_1D }}=800$; spikes (d): $\phi_{0}=3.0 \mathrm{~J} / \mathrm{cm}^{2}$, $\left.N_{\text {eff_1D }}=400\right]$. The red double arrow indicates the direction of the polarization, which coincides with the scan direction. 


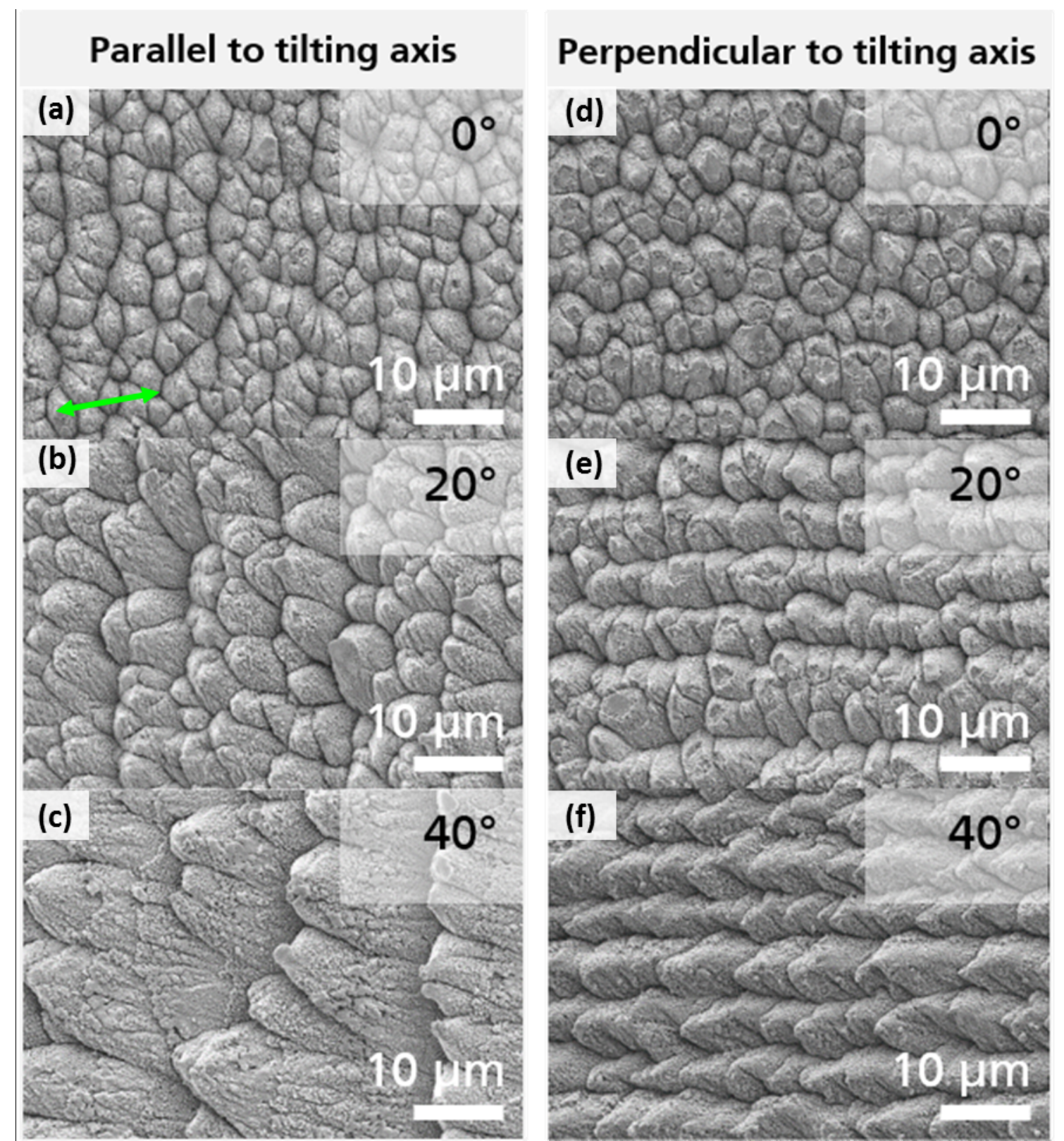

Fig. 4. Top-view $\left(0^{\circ}\right)$ SEM micrographs of spike structures on $42 \mathrm{CrMo} 4$ steel after ps-laser irradiation [Processing conditions: $\lambda=532 \mathrm{~nm}, \tau=9 \mathrm{ps}, f=1 \mathrm{MHz} ; \phi_{0}=0.13-0.17 \mathrm{~J} / \mathrm{cm}^{2}$; $\left.N_{\text {eff_-1D }} \approx 2.5-3.5 \times 10^{4}\right]$ at an angle of incidence of $0^{\circ}\left[(\mathrm{a})\right.$ and (d)], $20^{\circ}\left[(\mathrm{b})\right.$ and (e)], and $40^{\circ}[(\mathrm{c})$ and (f)]. The scan direction was parallel [(a), (b), and (c)] and perpendicular [(d), (e) and (f)] to the tilting axis of the sample. The green double arrow indicates the direction of the laser beam polarization. 

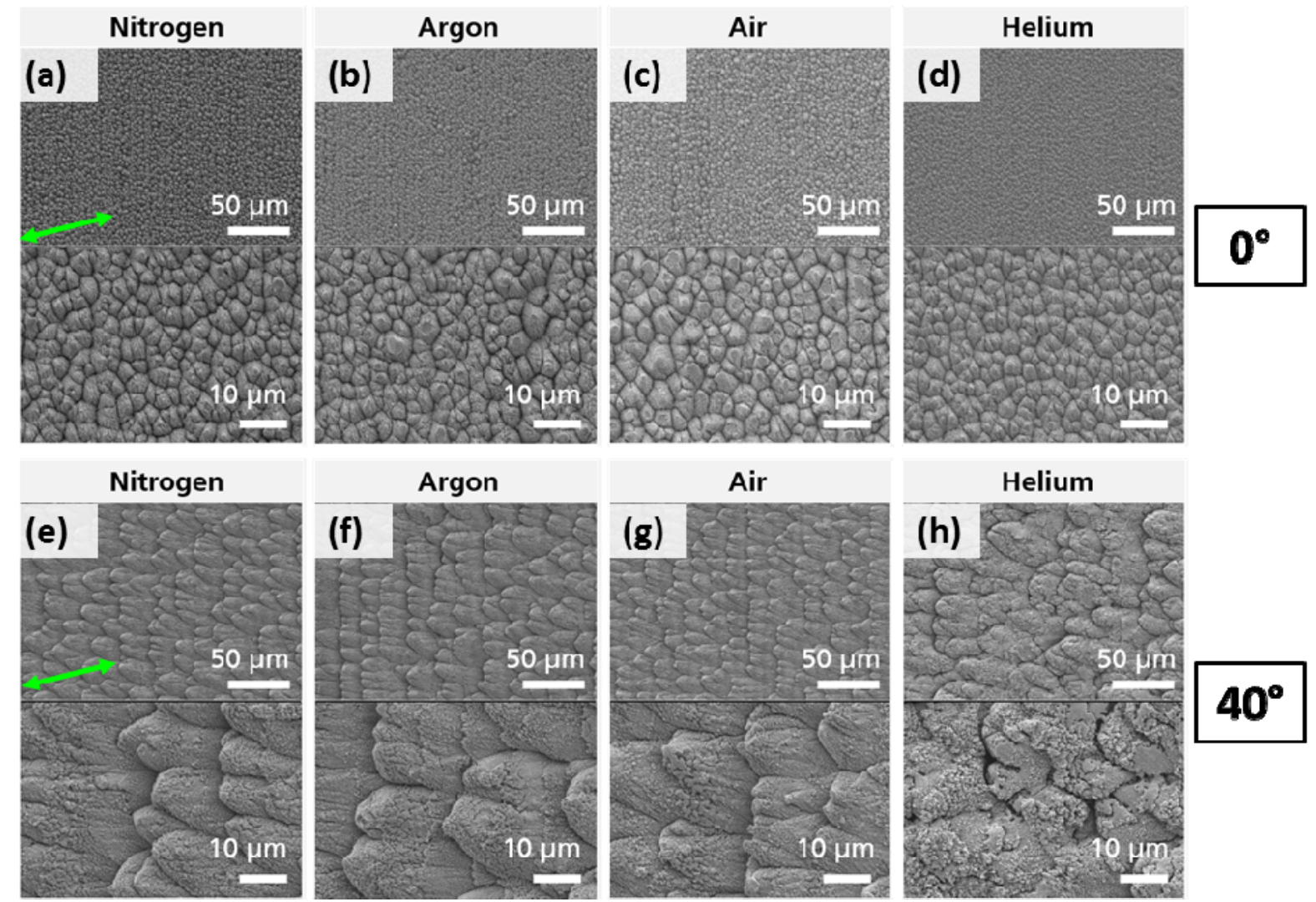

Fig. 5. Top-view $\left(0^{\circ}\right)$ SEM micrographs of spike structures on $42 \mathrm{CrMo} 4$ steel after ps-laser irradiation [Processing conditions: $\lambda=532 \mathrm{~nm}, \tau=9 \mathrm{ps}, f=1 \mathrm{MHz} ; \phi_{0}=0.13-0.17 \mathrm{~J} / \mathrm{cm}^{2}$; $\left.N_{\text {eff_1D }} \approx 2.5-3.3 \times 10^{4}\right]$ at different angles of incidence [(a)-(d): $0^{\circ}$; (e)-(h): $40^{\circ}$ ] and different gas atmospheres [(a) and (e): nitrogen; (b) and (f): argon; (c) and (g): air; (d) and (h): helium]. Note the different magnifications. The green double arrow indicates the direction of the laser beam polarization. 


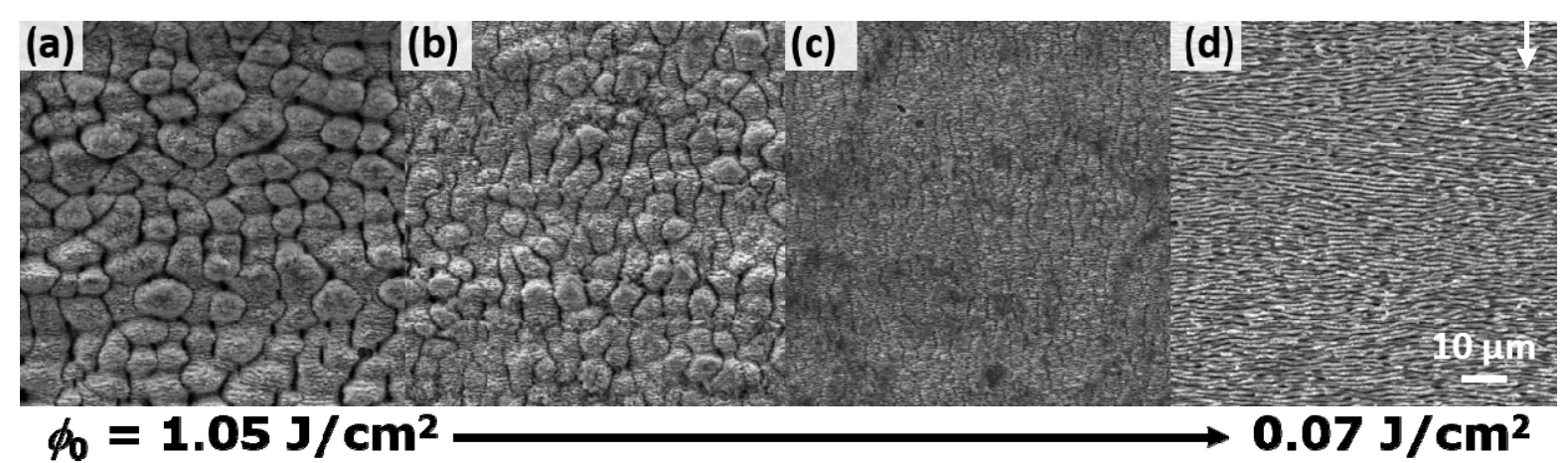

Fig. 6. Top-view SEM micrographs of four different surface regions within a gradient structures fs-laser processed on $42 \mathrm{CrMo} 4$ steel [Processing conditions: $\lambda=1026 \mathrm{~nm}, \tau=170 \mathrm{fs}, f=1 \mathrm{kHz}$; $N_{\text {eff_1D }}=369 ; \phi_{0}=1.05 \mathrm{~J} / \mathrm{cm}^{2}$ (a) $-0.07 \mathrm{~J} / \mathrm{cm}^{2}$ (d)] by variation of $\phi_{0}$. Spikes structured at the maximum peak fluence (a), transition from spikes to grooves as fluence is reduced (b), further reduction in fluence leads to the formation of grooves (c). LSFL-ripples are formed at the lowest fluence applied (d). The direction of the laser polarization is vertical, as indicated with white double arrow, while the scan direction is horizontal. 


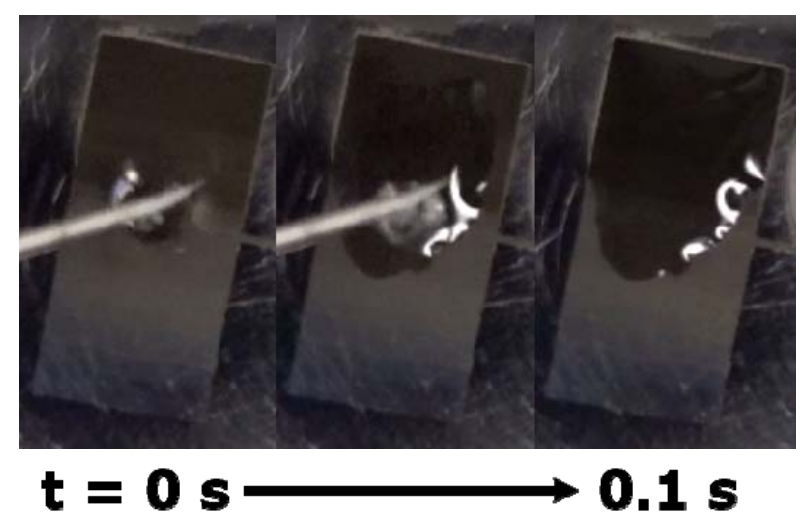

Fig. 7. Sequence of top-view photographs $(t=0-0.1 \mathrm{~s} ; \Delta t=0.05 \mathrm{~s})$ demonstrating the unidirectional fluid transport of a droplet of water $(8 \mu \mathrm{l})$, placed in the centre of a fs-laser processed gradient structure at $t=0$ [processing conditions: $N_{\text {eff_1D }}=369 ; \phi_{0}=1.05 . \mathrm{J} / \mathrm{cm}^{2}$, (top region, associated with spikes) $-0.07 . \mathrm{J} / \mathrm{cm}^{2}$, (bottom region, associated with LSFL-ripples); area: $8 \times 15 \mathrm{~mm}^{2}$; processed 1 day before water transport experiments] on $42 \mathrm{CrMo} 4$ steel. 


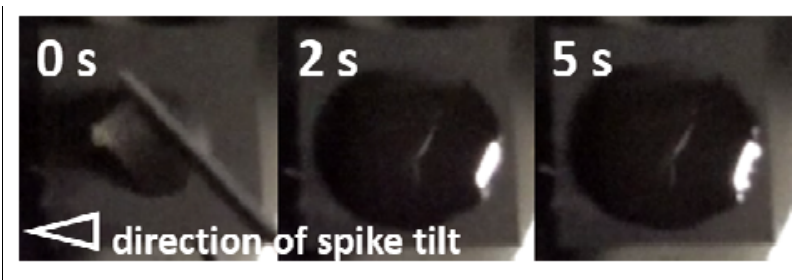

Fig. 8. Sequence of top-view photographs $(t=0,2$ and $5 \mathrm{~s}$ after the oil droplet was placed on the surface) demonstrating the omnidirectional fluid transport of a droplet of engine oil $(8 \mu 1$, Shell Rimula), placed in the centre of a surface of fs-laser processed tilted spikes [compare Fig. 4(f); irradiation conditions: angle of incidence $40^{\circ}$, scanning perpendicular to the tilting axis, $\phi_{0}=0.13$ $\mathrm{J} / \mathrm{cm}^{2}, N_{\text {eff_1D }} \approx 3.3 \times 10^{4}$; processed area: $\left.5 \times 5 \mathrm{~mm}^{2}\right]$ on $42 \mathrm{CrMo} 4$ steel. 

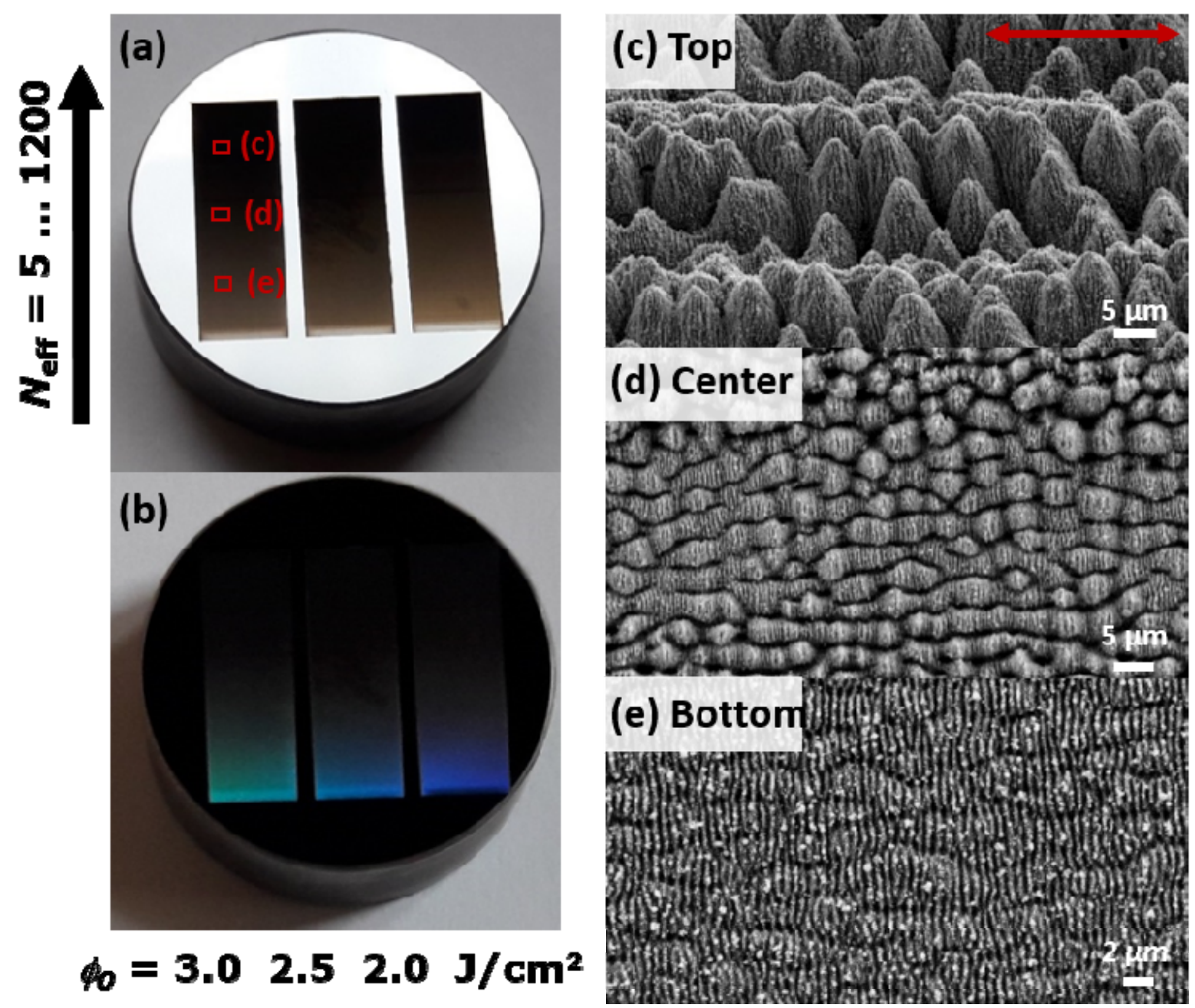

Fig. 9. Top-view (a) and tilted-view (b) photographs of the fs-laser laser processed gradient structures on $42 \mathrm{CrMo} 4$ steel samples (diameter $24 \mathrm{~mm}$ ) [Processing conditions: $\lambda=790 \mathrm{~nm}$, $\tau=30 \mathrm{fs}, f=1 \mathrm{kHz} ; N_{\text {eff_1D }}=5$ (bottom) -1200 (top); $\phi_{0}=3.0 \mathrm{~J} / \mathrm{cm}^{2}$ (left area), $\phi_{0}=2.5 \mathrm{~J} / \mathrm{cm}^{2}$ (central area) $\phi_{0}=2.0 \mathrm{~J} / \mathrm{cm}^{2}$ (right area)]. SEM micrographs of the gradient structure on the left, corresponding to the red rectangles in (a): (c) spikes $\left(60^{\circ}\right.$ tilted view); (d) grooves $\left(0^{\circ}\right)$ and (e) LSFL-ripples $\left(0^{\circ}\right)$. Note the different magnifications used in SEM. The scan direction and the laser beam polarization (indicated by the red arrow) are both horizontal.

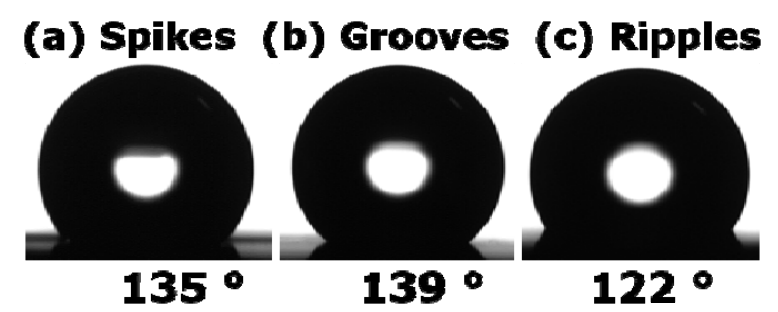

Fig. 10. Side-view photographs of $4 \mu \mathrm{l}$ droplets of deionized water on the fs-laser processed [Processing conditions: $\lambda=790 \mathrm{~nm}, \tau=30 \mathrm{fs}, f=1 \mathrm{kHz}, \phi_{0}=3.0 \mathrm{~J} / \mathrm{cm}^{2}$ ] gradient structures on $42 \mathrm{CrMo} 4$ steel and their contact angles: (a) top region, associated with spikes; (b) central region associated with grooves; (c) bottom region, associated with LSFL-ripples on 42CrMo4 steel. 


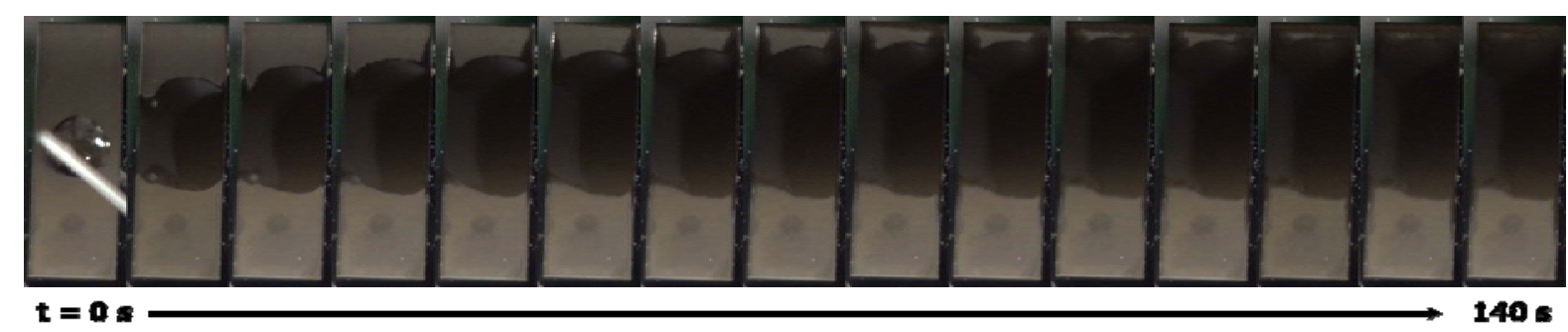

Fig. 11. Sequence of top-view photographs $(t=0-140 \mathrm{~s} ; \Delta t=10 \mathrm{~s})$ demonstrating the unidirectional fluid transport of a droplet of engine oil ( $8 \mu 1$, Shell Rimula), placed in the centre of a fs-laser processed gradient structure at $t=0-140 \mathrm{~s}$ [Processing conditions: $\lambda=790 \mathrm{~nm}, \tau=30$ fs, $f=1 \mathrm{kHz}, \phi_{0}=3.0 \mathrm{~J} / \mathrm{cm}^{2}, N_{\text {eff_1D }}=1200$ (top region, associated with spikes) -5 (bottom region, associated with LSFL-ripples); processed area: $\left.5 \times 15 \mathrm{~mm}^{2}\right]$ on $42 \mathrm{CrMo} 4$ steel. 


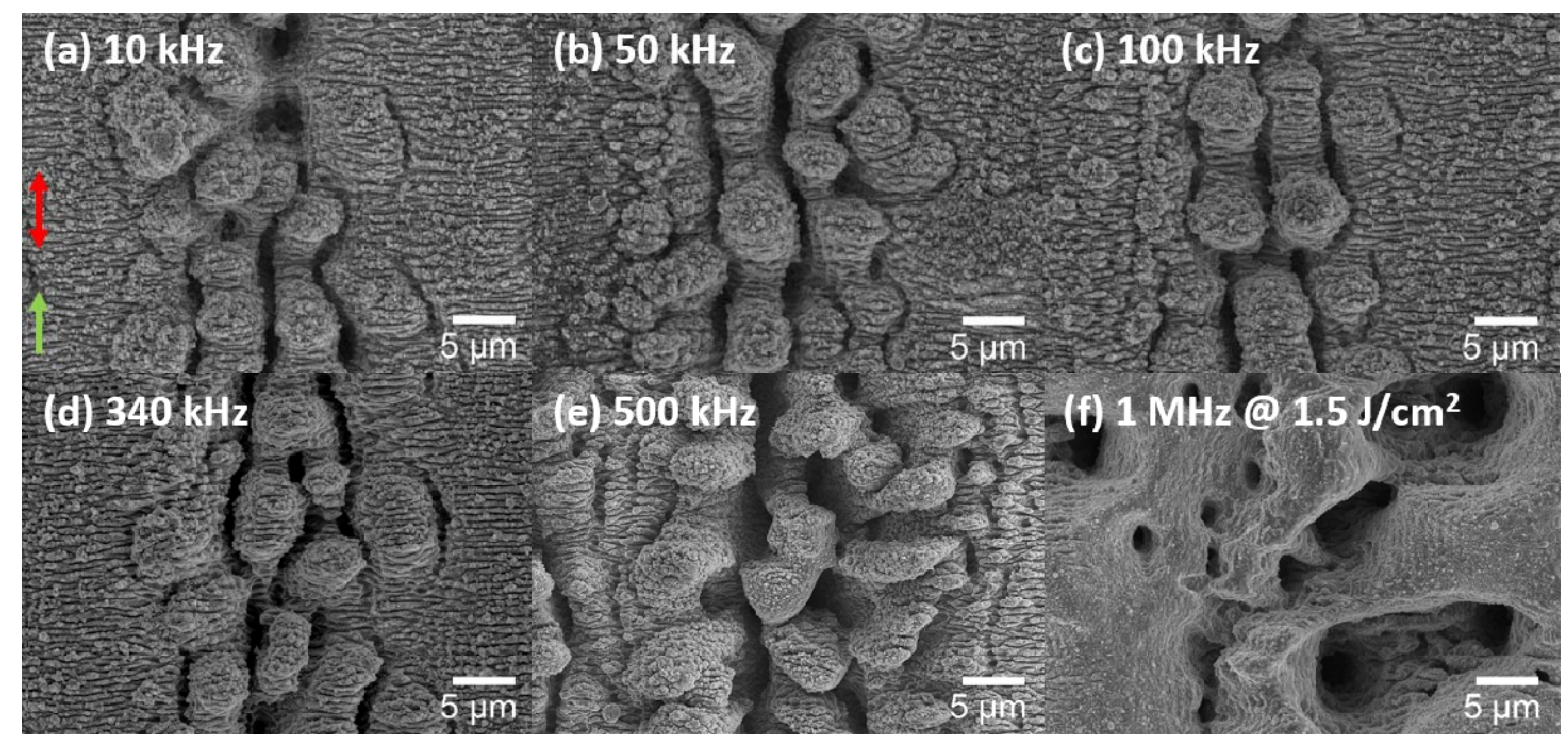

Fig. 12. SEM micrographs of spikes formed on $42 \mathrm{CrMo} 4$ steel surfaces upon fs-laser processing $\left[\lambda=1030 \mathrm{~nm}, \tau=500 \mathrm{fs}, N_{\text {eff }} 1 \mathrm{D}=400 ; \phi_{0}=2.0(\mathrm{a}-\mathrm{e})\right.$ and $1.5 \mathrm{~J} / \mathrm{cm}^{2}$ (f) $]$ at different repetition rates ranging from $10 \mathrm{kHz}$ (a) to $1 \mathrm{MHz}$ (f). The scan direction and the laser beam polarization are indicated by the green and red arrows in (a), respectively.

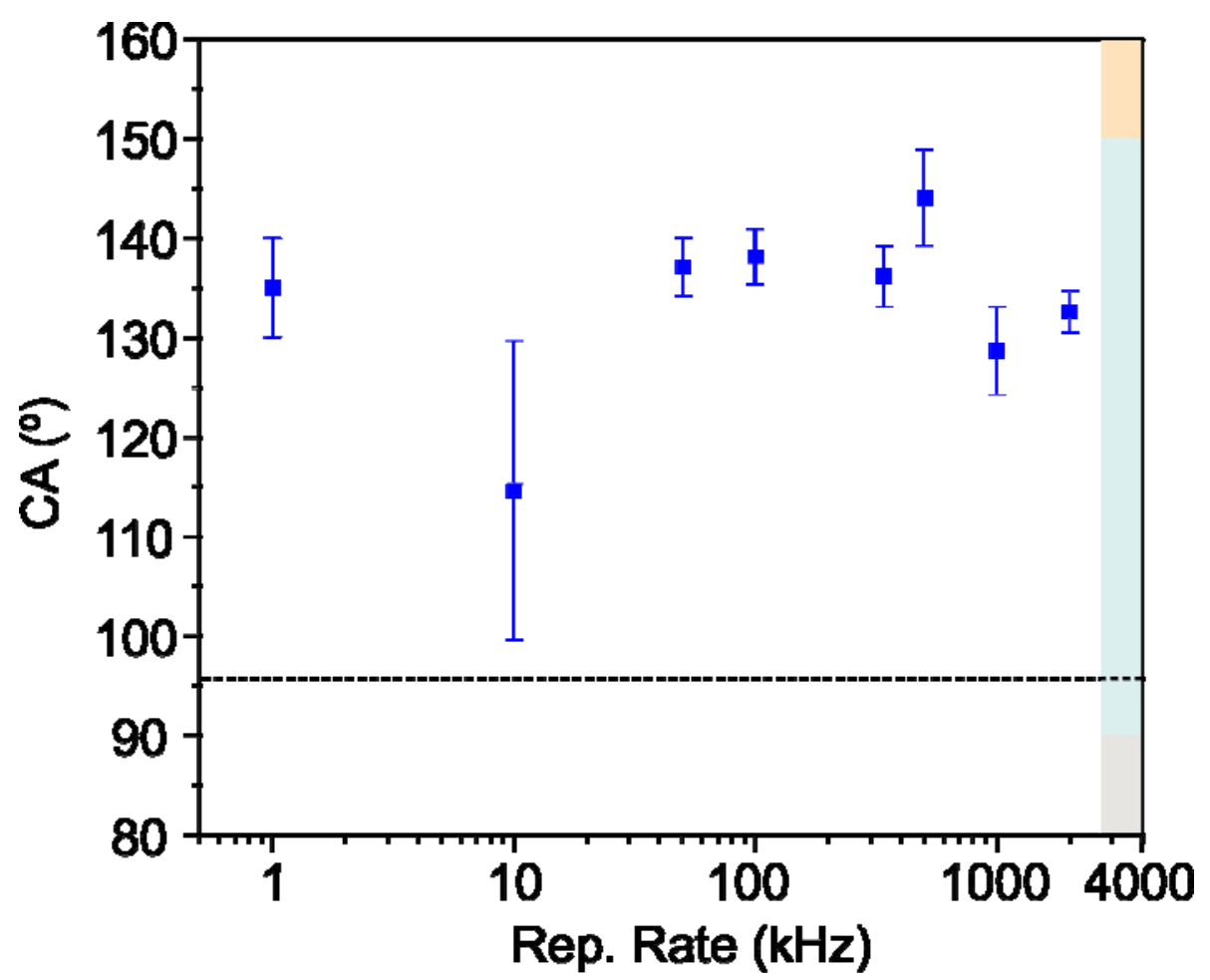

Fig. 13. Plot of the contact angle (CA) of a $4 \mu$ d droplet of deionized water versus the laser pulse repetition rate $\left[\lambda=1030 \mathrm{~nm}, \tau=500 \mathrm{fs} ; N_{\text {eff_1D }}=400 ; \phi_{0}=2.0 \mathrm{~J} / \mathrm{cm}^{2}(1-500 \mathrm{kHz})\right.$ and $1.5 \mathrm{~J} / \mathrm{cm}^{2}$ $(\geq 1000 \mathrm{kHz})]$. Error bars correspond to uncertainty in the measurement obtained via software analysis. The dotted horizontal line at $95.7^{\circ}$ corresponds to the CA of the pristine (polished) steel surface. On the right part, colour-shaded areas indicate regions of hydrophilicity (grey, $\mathrm{CA}<90^{\circ}$ ), hydrophobicity (blue, $90^{\circ}<\mathrm{CA}<150^{\circ}$ ) and super-hydrophobicity (yellow, $150^{\circ}<\mathrm{CA}$ ). The data point at $1 \mathrm{kHz}$ was taken from Tab. 2 (Spikes, $\lambda=790 \mathrm{~nm}, \tau=30 \mathrm{fs}, N_{\text {eff_1D }}=400$; $\phi_{0}=2.0 \mathrm{~J} / \mathrm{cm}^{2}$ ). 


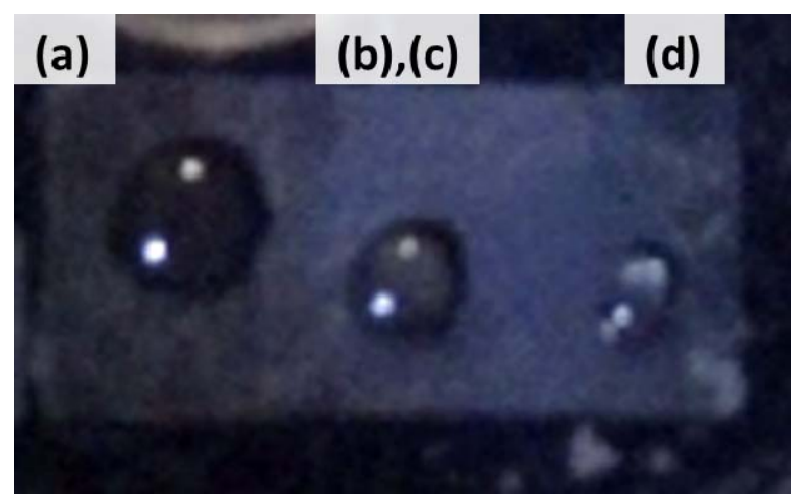

Fig. 14. Top-view photograph of $4 \mu 1$ droplets of deionized water on the fs-laser processed gradient structures on $42 \mathrm{CrMo} 4$ steel taken 14 days after irradiation [Processing conditions: $\lambda=1026 \mathrm{~nm}, \tau=170 \mathrm{fs}, f=1 \mathrm{kHz}, N_{\text {eff_1D }}=369 ; \phi_{0}=1.05 \mathrm{~J} / \mathrm{cm}^{2}$, (left, associated with spikes) 0.07. $\mathrm{J} / \mathrm{cm}^{2}$, (right, associated with LSFL-ripples); area: $\left.8 \times 15 \mathrm{~mm}^{2}\right]$ : (a) left region, associated with spikes; (b/c) central region associated with grooves; (d) right region, associated with LSFLripples. (a-d) associated with regions previously shown in Fig. 6. 